\title{
INTOXICACIÓN POR NITRATOS EN UNA CUADRA DE CABALLOS:
} DESCRICIÓN DE UN CASO

\section{NITRATE INTOXICATION IN A HORSE STABLES: CASE REPORT}

* Gómez Cisneros, D. ${ }^{1,2,4,5}$; Tapia, J. ${ }^{1,5}$; Magaña, A. ${ }^{3}$; Pile, E. ${ }^{1,4}$; Fores, P. ${ }^{2}$

${ }^{1}$ Departamento de Clínicas y Cirugías, Facultad de medicina veterinaria, Universidad de

Panamá, Ciudad de Panamá. ${ }^{2}$ Departamento de Medicina y Cirugía Animal, Facultad de veterinaria, Universidad Complutense de Madrid, Madrid, España. ${ }^{3}$ Centro de Diagnóstico e Investigación Veterinaria (CEDIIVET), Ciudad de Panamá, Panamá. ${ }^{4}$ Investigador, Secretaría nacional de ciencias, tecnología e innovación (SENACYT), Gobierno de Panamá, Ciudad de Panamá. ${ }^{5}$ Médico Veterinario, Complejo Hospitalario Veterinario de Corozal, Universidad de Panamá.*Autor de correspondencia: david_gomez09@hotmail.com

\section{RESUMEN}

Exponemos el cuadro observado en na cuadra de 11 caballos que mostraron sintomatología aguda con intoxicación. Los caballos consumían alimento concentrado y heno, pero por motivos estacionales, se alimentaron con pasto de Digitaria swazilandensis. Se dejaron en ayunas los caballos durante 48 horas, con agua ad libitum. Se instauró un tratamiento sintomático y todos los caballos mejoran. Se introduce el pasto de nuevo y se produce recaída y muerte de 3 caballos a los que se les realiza necropsia, donde se confirma la sospecha de intoxicación con afectación digestiva, hepática y encefálica. Se recogen muestras del alimento concentrado, forraje y agua para análisis toxicológico, bacteriológico y fúngico, y se evidencia elevadas concentraciones de nitratos en el pasto. La intoxicación por nitratos es infrecuente en caballos y para un diagnóstico correcto es necesario realizar un estudio anatomopatológico y determinación de los niveles de nitratos de las posibles fuentes de contaminación.

Palabras clave: caballos, nitratos, intoxicación, estudio anatomopatológico. 


\begin{abstract}
We expose a stable of 11 horses that showed acute symptomatology compatible intoxication. The horses consumed concentrated feed and hay, but for seasonal reasons, were fed with grass of Digitaria swazilandensis. The horses were fasted for 48 hours with water ad libitum. Symptomatic treatment was instated and all horses improved. The grass is introduced again and there is relapse and death of 3 horses, that are necropsied, where it confirms the suspicion of intoxication with digestive, hepatic and encephalic affectation. Samples of the concentrate feed, forage and water are collected for toxicological bacteriological and fungal analysis and high concentrations of nitrates in the pasture are evidenced. Nitrate intoxication is uncommon in horses and for a correct diagnosis it is necessary to perform a pathological study and determination of nitrate levels of possible sources of contamination.
\end{abstract}

Keywords: Horses, nitrates, intoxication, anatomopathological study.

\title{
INTRODUCCIÓN
}

La intoxicación con nitratos y nitritos generalmente se desarrollan después de consumir pasto y agua que contienen elevadas concentraciones de nitratos o por consumo accidental de los animales de sustancias que contienen nitrato como algunos fertilizantes (Kahn 2005).

Los rumiantes son especialmente susceptibles, porque la flora ruminal reduce el nitrato a nitrito, siendo este último más tóxico que el nitrato (Issi y col 2008). Entre los animales monogástricos los caballos son los más susceptibles, porque tienen un ciego que actúa como rumen convirtiendo el nitrato en nitrito (pero no en igual medida que los rumiantes). Los nitratos que se ingieren pueden digerirse directamente e irritar la mucosa gastrointestinal y producir dolor abdominal y diarrea. El nitrito como tal causa metahemoglobinemia que se absorbe por la mucosa gastrointestinal, en consecuencia el tejido no recibe suficiente oxígeno y se desarrolla hipoxia anémica (Schneider 1998). La intoxicación por nitratos y nitritos es rara en caballos y existe poca bibliografía e información de intoxicaciones en esta especie.

En este caso se describen los signos clínicos, diagnóstico, tratamiento y hallazgos postmortem de una cuadra de caballos en la que se sospechó intoxicación por nitratos.

\section{DESCRIPCIÓN DEL CASO}

Se expone el cuadro de intoxicación sufrido en una cuadra de 11 caballos de diferentes edades, razas y sexo que fueron remitidos al Complejo Hospitalario Veterinario de Corozal- 
Universidad de Panamá (SIADVET) por que mostraron sintomatología aguda compatible con intoxicación por nitratos. Tres de los afectados mueren 96 horas después.

Los caballos estaban estabulados y se alimentaban con concentrado ( $5 \mathrm{~kg}$ ) y heno, pero en el mes de mayo con el cambio de estación seca a estación lluviosa, se decide alimentar los caballos con pasto de Digitaria swazilandensis (el cual no se cortaba desde hace 8 meses). No hay historia previa de fertilización con compuestos nitrogenados ni herbicidas. El primer caso tuvo aparición súbita 4 días antes, pero muere a las pocas horas.

Primero se realizó la exploración en 8 caballos en los que se observa deshidratación leve, taquicardia, taquipnea, sudoración, decoloración de la mucosa gingival, temblores, ataxia grave, temperatura corporal alta $\left(39.5-40{ }^{\circ} \mathrm{C}\right)$, dolor abdominal con poca respuesta a la analgesia. Uno de los caballos estaba en decúbito con tremores, nistagmo y convulsiones. Tres de los caballos de la cuadra no mostraban aun sintomatología (Figura 1).
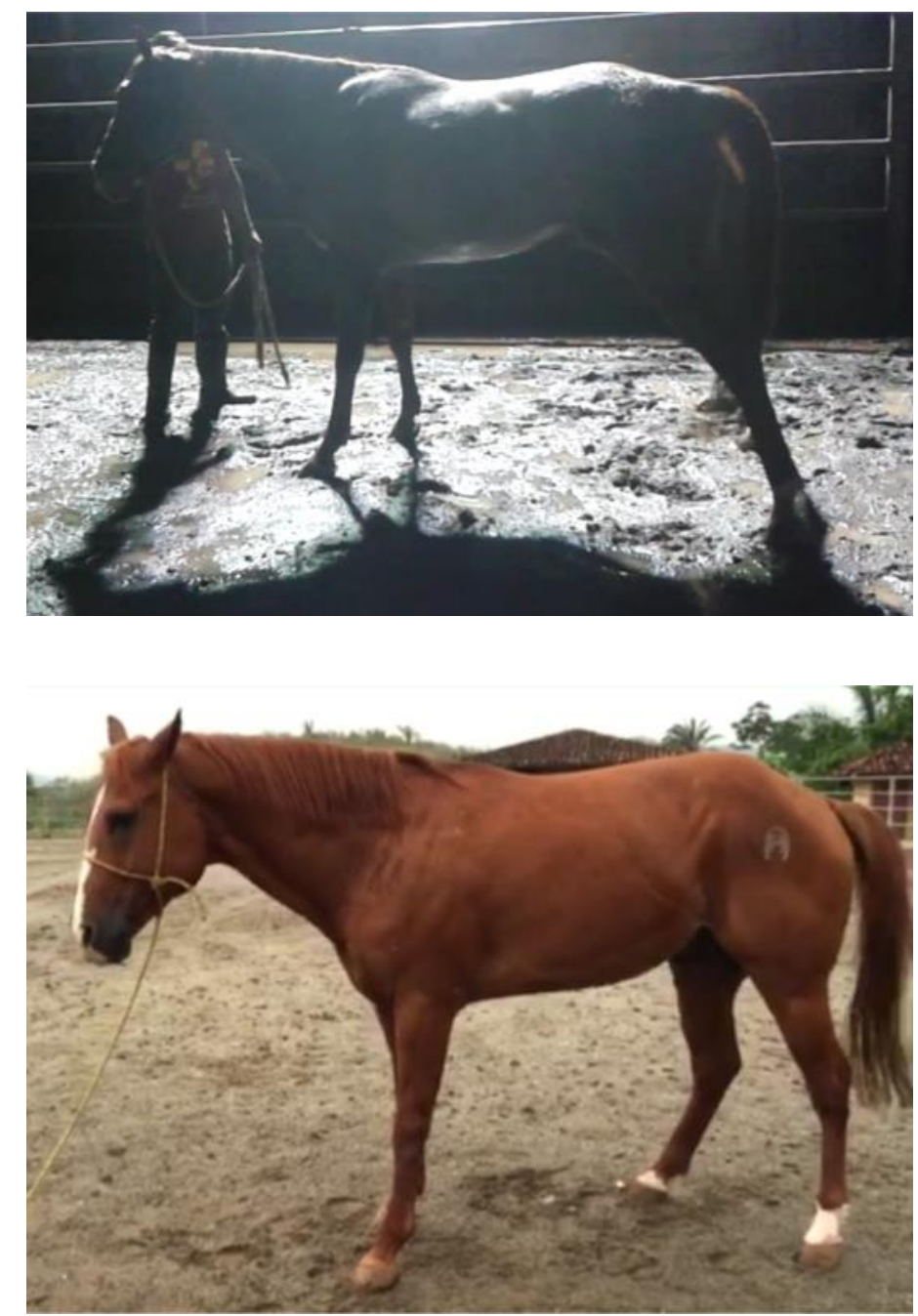

Figura 1: caballo afectado, con ataxia evidente y base de sustentación amplia de las extremidades. 
Se realiza hematología de todos los caballos de la explotación y se observan en 7 de ellos una disminución de la $\mathrm{Hb}$, Htc y recuento de glóbulos rojos y blancos, así como neutrofilia y linfopenia. La bioquímica sanguínea muestra fundamentalmente alteración de las enzimas hepáticas (GGT aumentada en todos) y bilirrubinemia en 8 de los caballos (Tabla 1).

\section{Tabla 1: Hematología y bioquímica sanguínea}

\begin{tabular}{|c|c|c|c|c|c|c|c|c|c|c|c|c|}
\hline & REFERENCIA & 1 & 2 & 3 & 4 & 5 & 6 & 7 & 8 & 9 & 10 & 11 \\
\hline \multicolumn{13}{|c|}{ Hematología } \\
\hline $\mathrm{RBC}$ & $7-13.1\left(\mathrm{x} 10^{3} / \mathrm{mm}^{3}\right)$ & $6.28 \downarrow$ & 7.55 & $6.55 \downarrow$ & $6.07 \downarrow$ & $5.87 \downarrow$ & 7.58 & $6.07 \downarrow$ & $6.6 \downarrow$ & 8.4 & $5.3 \downarrow$ & 7.7 \\
\hline Hemoglobina & $11-19.1(\mathrm{~g} / \mathrm{dL})$ & $9.7 \downarrow$ & 11.7 & $10.7 \downarrow$ & $10.4 \downarrow$ & $9.5 \downarrow$ & 12.9 & $9.7 \downarrow$ & 11.5 & 13.6 & $8.2 \downarrow$ & 12.1 \\
\hline Hematocrito & $32-52(\%)$ & $26.5 \downarrow$ & 32.8 & 33.6 & $31.5 \downarrow$ & $30.4 \downarrow$ & 37.1 & $29.1 \downarrow$ & 35.4 & 40.9 & $24.9 \downarrow$ & 34.4 \\
\hline WBC & $6-12.5\left(\mathrm{x} 10^{3} / \mathrm{mm}^{3}\right)$ & 8.6 & $4.9 \downarrow$ & $6.4 \downarrow$ & $5.9 \downarrow$ & $5.7 \downarrow$ & $5 \downarrow$ & $6.4 \downarrow$ & 7.5 & 7.5 & $4 \downarrow$ & 11.2 \\
\hline Neutrófilos & $25-70(\%)$ & $78 \uparrow$ & $74 \uparrow$ & $76 \uparrow$ & $80 \uparrow$ & $85 \uparrow$ & $77 \uparrow$ & $78 \uparrow$ & $71 \uparrow$ & $74 \uparrow$ & $77 \uparrow$ & $84 \uparrow$ \\
\hline Linfocitos & $15-60(\%)$ & $7 \downarrow$ & 17 & 15 & $14 \downarrow$ & $8 \downarrow$ & $14 \downarrow$ & 16 & 17 & 17 & 14 & $9 \downarrow$ \\
\hline Monocitos & $1.1-10(\%)$ & 9 & 6 & 6 & 4 & 5 & 7 & 4 & 7 & 6 & 6 & 6 \\
\hline Eosinófilos & $1.1-10(\%)$ & 6 & 3 & 3 & 2 & 2 & 2 & 2 & 5 & 3 & 3 & 1 \\
\hline Plaquetas & $90-350\left(\times 10^{3} / \mathrm{mm}^{3}\right)$ & 234 & 210 & 145 & 157 & 141 & 109 & 131 & 170 & 127 & 113 & 170 \\
\hline \multicolumn{13}{|l|}{ Bioquímica } \\
\hline Proteínas totales & $5.2-7.9(\mathrm{~g} / \mathrm{dL})$ & 5.5 & 6.6 & 7 & 5.8 & 6.7 & 6.4 & 5.7 & 6.6 & 6.3 & 7.1 & 6 \\
\hline Albumina & $2.6-3.7(\mathrm{~g} / \mathrm{dL})$ & 3.5 & $4.1 \uparrow$ & $4.5 \uparrow$ & 2.9 & $4.5 \uparrow$ & 3.2 & 2.7 & $3.7 \uparrow$ & $4.3 \uparrow$ & $4.2 \uparrow$ & $4.2 \uparrow$ \\
\hline Globulinas & $2.6-4.0(\mathrm{~g} / \mathrm{dL})$ & $2 \downarrow$ & $2.5 \downarrow$ & 2.5 & 2.9 & 2.2 & 3.2 & 3 & 2.9 & $2 \downarrow$ & 2.9 & 1.8 \\
\hline Bil. Total & $0-2(\mathrm{mg} / \mathrm{dL})$ & 0.3 & 0.3 & 0.3 & $4.5 \uparrow$ & 0.3 & $3.5 \uparrow$ & $2.3 \uparrow$ & 0.4 & 0.3 & 0.4 & 0.2 \\
\hline Bil. Indirecta & $0.2-2(\mathrm{mg} / \mathrm{dL})$ & 1.1 & $3 \uparrow$ & 1.9 & $4.1 \uparrow$ & 1.8 & $3.1 \uparrow$ & $2.1 \uparrow$ & $2.4 \uparrow$ & 1.9 & $2.2 \uparrow$ & 1.1 \\
\hline Bil. Directa & $0-0.4(\mathrm{mg} / \mathrm{dL})$ & $1.4 \uparrow$ & $3.3 \uparrow$ & $2.2 \uparrow$ & 0.4 & $2.1 \uparrow$ & 0.4 & 0.2 & $2.8 \uparrow$ & $2.2 \uparrow$ & $2.6 \uparrow$ & $1.3 \uparrow$ \\
\hline AST & $226-336(\mathrm{U} / \mathrm{L})$ & 297 & $222 \downarrow$ & 258 & $479 \uparrow$ & 189 & $1125 \uparrow$ & 198 & 287 & 235 & 264 & 250 \\
\hline GGT & 4-13 (U/L) & $24 \uparrow$ & $27 \uparrow$ & $19 \uparrow$ & $34 \uparrow$ & $16 \uparrow$ & $64 \uparrow$ & $17 \uparrow$ & $18 \uparrow$ & $22 \uparrow$ & $30 \uparrow$ & $13 \uparrow$ \\
\hline Urea & $10-24(\mathrm{mg} / \mathrm{dL})$ & 21 & 11 & 15 & $24 \uparrow$ & $30 \uparrow$ & 21 & 11 & 18 & $25 \uparrow$ & 11 & 18 \\
\hline Creatinina & $1.2-1.9(\mathrm{mg} / \mathrm{dL})$ & $2.2 \uparrow$ & 1.5 & 1.4 & 1.8 & $2 \uparrow$ & 1.9 & 1.5 & 1 & $2 \uparrow$ & 1.5 & 1.6 \\
\hline
\end{tabular}

Como se sospecha una intoxicación, se decide mantener en ayunas de concentrado y pasto durante 48 horas a todos los caballos, con agua ad libitum (pero recolectada de otro pozo) y se instaura un tratamiento sintomático basado en: Fluidoterapia (solución salina fisiológica $(0.9 \%)$ intravenosa), lavado naso-gástrico y carbón activado, atropina $(0.02 \mathrm{mg} / \mathrm{kg})$, flunixin meglumine $(1.1 \mathrm{mg} / \mathrm{kg})$, como analgésico y para evitar el shock endotóxico, dexametasona $(0.1 \mathrm{mg} / \mathrm{kg})$, clorfeniramina $(1 \mathrm{mg} / \mathrm{kg})$, y protector hepático (Hepatofactor $\mathrm{A} \circledast)$.

Se envían al laboratorio muestras de pasto (nitratos y nitritos), agua (coliformes totales, sulfatos, turbidez, $\mathrm{pH}$, sulfatos, metales, nitratos y nitritos) y concentrado (recuento de hongos y sustancias nitrogenadas) para su análisis.

Todos los caballos mejoran a las 48 horas y a las 72 horas se decide dar pequeñas cantidades de pasto. A las 96 horas 5 caballos presentan de nuevo sintomatología; 3 que no habían 
mostrado síntomas previamente y 2 que sufren una recaída. Tres de ellos mueren y se realiza necropsia 4 horas después.

En los hallazgos post-mortem, se observó macroscópicamente mucosa muy congestiva (rojo oscuro), en la cavidad torácica abundante liquido rojo brillante, zonas de infarto y equimosis, la mucosa del estómago e intestino estaban edematizadas y zonas con congestión y erosión, afectación hepática y congestión de los vasos sanguíneos del cerebro (Figura 2).
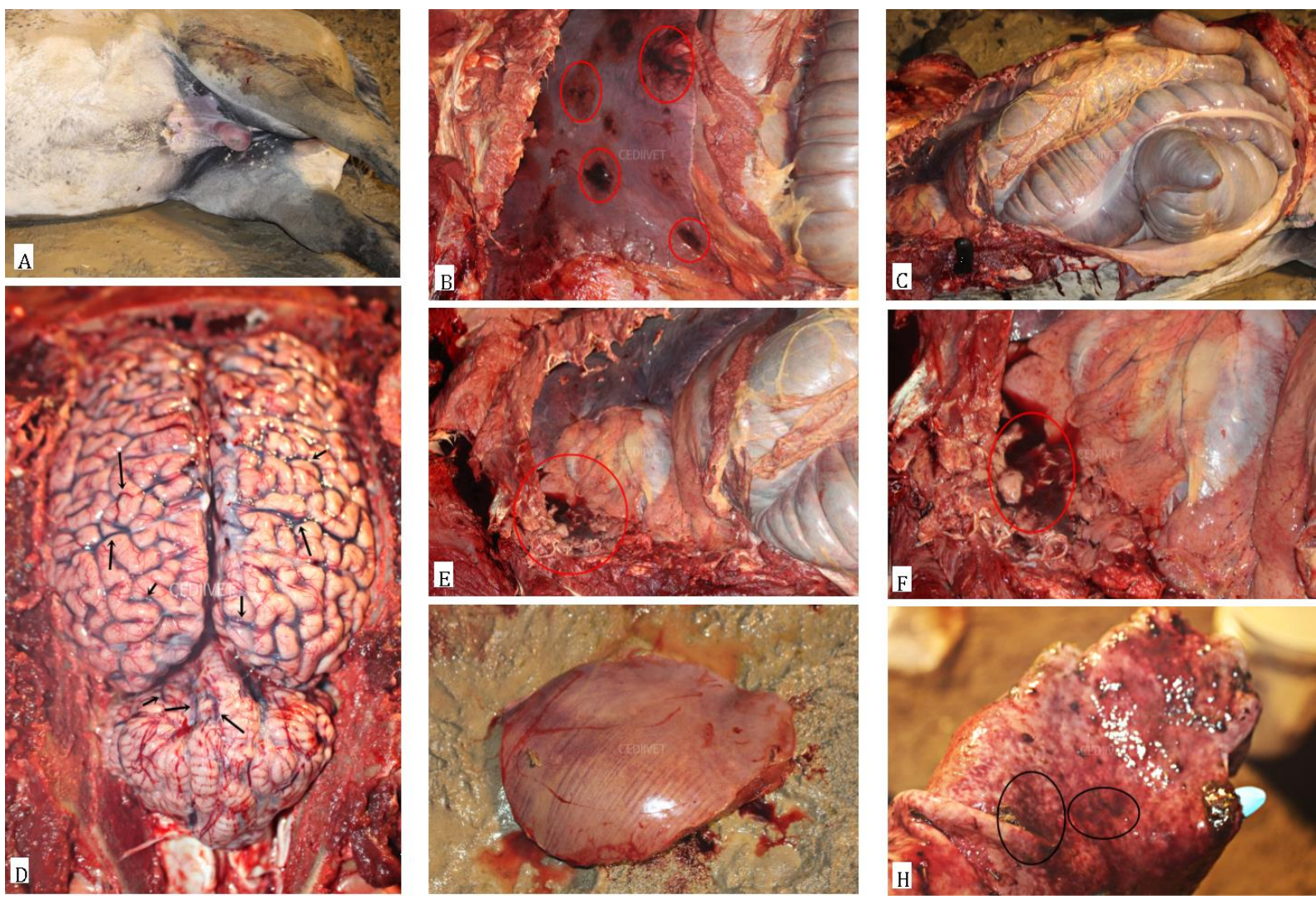

Microscópicamente se observó marcada pérdida de las vellosidades intestinales, zonas de fibrosis, cambios de la apetencia tintorial e infiltrado linfocítico en la membrana basal, a nivel hepático se observa necrosis hepática centrolobulilllar indicativa de la existencia de un posible agente tóxico, vacuolización en el sistema nervioso (médula oblonga). La tinción de Gram muestra bacilos Gram positivos en grupos y adheridos a la mucosa intestinal.

Por prevención, se decide mantener nuevamente en ayunas de pasto a todos los caballos hasta tener los resultados de los análisis y se continua con el tratamiento sintomático. Tras instaurar estas medidas los caballos presentaron mejoría y se recuperaron en su totalidad.

Los resultados de los análisis (Tabla 2) muestran elevadas concentraciones de nitratos en el pasto, y también de elevadas concentraciones de compuestos nitrogenados en el concentrado. 
El agua de ambos pozos esta dentro de los parámetros normales. El recuento de hongos, se encontraba dentro de los valores estándares.

\section{Tabla 2: Análisis laboratorio}

\begin{tabular}{|lccc|}
\hline \multicolumn{1}{l}{ Muestras } & Nitratos $(\mathbf{m g} / \mathbf{k g})$ & Nitritos $(\mathbf{m g} / \mathbf{k g})$ & Nitrógeno amoniacal $(\mathbf{m g} / \mathbf{k g})$ \\
Pasto & & & 238 \\
Parcela 1 & 668 & $<0.001$ & 127.2 \\
Parcela 2 & 266 & $<0.001$ & 202.8 \\
Parcela 4 & 410.0 & 8 & 147 \\
Agua & 750 & $<0.001$ & $<0.001$ \\
Pozo 1 & 0.2 & $<0.001$ & $<0.001$ \\
Pozo 2 & 0.04 & $<0.001$ & 200 \\
Concentrado & 850 & $<0.001$ & \\
\hline
\end{tabular}

Dosis toxica de nitratos (Lorge y col 1996): 61-152 g por animal.

\section{DISCUSIÓN}

La intoxicación por nitratos es muy poco frecuente y está poco descrita en caballos (Lorgue y col. 1996; Issi y col 2008; Hasan y col 2010). Inicialmente se pensó que la causa de intoxicación era el concentrado, pero a pesar de su alto nivel en los análisis se descartó, ya que el lote de concentrado fue distribuido en varias cuadras de la zona y no se reportaron casos de otros caballos intoxicados. Además, este hecho fue confirmado porque fue suspendido desde el día 1 y no intervino en la recaída y aparición de nuevos casos ocurrida 96h. después. El origen de la intoxicación fue el pasto, ya que el ofrecido a los caballos antes del día 1 y 96 horas después, era de la parcela 4 en la que los resultados del análisis mostro elevadas concentraciones de nitratos. Posteriormente se ofrece heno, comprado en otra cuadra cercana (48 horas después) y no volvieron a presentar sintomatología.

Se han descrito muchas plantas que almacenan nitratos y son capaces de producir intoxicación (Francis 2009), pero no hemos encontrado referencias sobre la Digitaria swazilandensis. Un factor importante es que llevaba mucho tiempo sin cortar y es posible que en ese periodo hubiera acumulado más nitratos de lo habitual, ya que se han descrito plantas que por condiciones ambientales como sequias y crecimiento rápidos por cambios de estación, son capaces de acumular nitratos (Lorgue y col 1996; Schmitz 1998; Nicholson 2007; Issi y col 2008).

La anatomía patológica revelo que el cuadro sufrido tenía un origen tóxico. La vía de ingreso más probable es la digestiva, ya que se muestra zonas de edema y fibrosis tanto del estómago 
como del intestino. Así mismo, la necrosis hepática centrolobulilllar indica que el agente tóxico interactuó con el hígado por vía sanguínea ya que los hepatocitos centrolobulillares son más sensibles ante tóxicos y la vacuolización en el sistema nervioso (médula oblonga) puede ser asociada al depósito de amonio. La muerte se asocia a un fallo respiratorio por compresión directa sobre el centro respiratorio (vacuolización y edema) o por un paro cardíaco debido a cambios metabólicos.

Las zonas de fibrosis y edema en la mucosa del estómago e intestino sugiere que el proceso puede tener más de 15 días de duración, por lo que la intoxicación se debió a la ingesta constante del toxico en la dieta. No se conoce realmente el mecanismo de conversión bacteriana de nitratos a nitritos en los equinos, sin embargo esta descrito que los nitratos causan erosión de la mucosa gástrica si son ingeridos en altas concentraciones (Lorgue y col 1996; Issi y col 2008; Pritam y col 2014). Esto, combinado la alteración que originan en la flora intestinal puede ser la causa de la intoxicación.

Es importante resaltar otros diagnósticos diferenciales en este caso, como intoxicaciones sustancias nitrogenadas de herbicidas, organofosforados y organoclorados. Las intoxicaciones fúngicas son más comunmente encontradas en caballos es por ello que es importante realizar analítica al respecto, pero en nuestro caso el recuento no fue significativo.

La intoxicación con nitratos es relativamente rara en la especie equina (Hassan y col 2010), pero en nuestro caso, por la sintomatología, los hallazgos post-mortem y las elevadas concentraciones de nitratos en pasto pueden ser la causa de intoxicación y muerte de los caballos. Hay una amplia variedad en el nivel tóxico de los nitratos, pero esas diferencias pueden ser atribuídas, en parte a cantidad ingeridas (Schmitz 1998; Francis 2009). Si se diagnóstica a tiempo y se retira la fuente puede ser tratada de forma satisfactoria.

\section{RELEVANCIA CLÍNICA}

La intoxicación por nitratos es infrecuente en caballos, pero debe estar entre los diagnósticos diferenciales cuando un grupo de caballos aparecen con síntomas de taquicardia y taquipnea, decoloración de mucosas, temblores, ataxia y convulsiones incluso muerte súbita. El diagnóstico se tiene que realizar en base a los síntomas, los hallazgos anatomopatológicos y la determinación de los niveles de nitratos de las posibles fuentes de contaminación.

\section{AGRADECIMIENTOS}


- Irving Monfante; asistencia en la parte microbiológica (Ministerio de Desarrollo Agropecuario).

- Angie Magaña; estudio anatomo-patológico (Centro de Diagnóstico e Investigación Veterinaria, CEDIIVET).

- Lic. Enzo de Gracia; estudio químico de pastos y agua (Centro de Investigaciones Químicas S.A, Laboratorio CIQSA).

- Eliecer Abrego, Pablo Castillo, Juan González, Harmodio Arcia; Estudiantes (Facultad de Medicina Veterinaria-Universidad de Panamá).

\section{BIBLIOGRAFIA}

Francis, G. Chapter 54: Disorders Caused by Toxicants. In: Large animal internal medicine: Fourth edition. El servier Inc. Westline Industrial Drive, Missouri. (2009). P. 1702

Hasan, H. O.; Ahmet, A.; Ilknur, U.; \& Engin, K. Nitrate Poisoning in Horses Associate With Ingestion of Forage and Alfalfa. Journal of Equine Veterinary Science. (2010) Vol 30, 3.

Issi, M; Gul, Y; Atessahin, A; Karahan, I. Acute nitrate poisoning in two cattle. Toxicol Environ Chem (2008); 90:135-140.

Lorgue, G.; Lechenet, J.; Rivie`re, A. Nitrates-Nitrites. Clinical Veterinary Toxicology. Oxford: Blackwell Science (1996); p.143-145.

Nicholson, SS. Nitrate and nitrite accumulating plants. In: Gupta RC, ed. Veterinary Toxicology, 1st ed. Amsterdam, The Netherlands: Elsevier B.V; (2007):876-877.

Pritam, S.; Vishal, Mahajan, Sunil, V.; Ashuma, M.; Partap, G. Toxicological and Pathological Review of Concurrent Occurrence of Nitrite Toxicity and Swine Fever in Pigs. Toxicology International (2014). Vol-21-2.

Schmitz, D. Chapter 20: Toxicologic problems. In: Equine Internal Medicine. Philadelphia: WB Saunders Company, (1998); p.1645-47.

Schneider NR. Nitrate and nitrite poisoning. In: Aiello SE, ed. The Merck Veterinary Manual, 8th ed. White House Station, NJ: Merck\&Co Inc; (1998): 2091-2094. 